\title{
Frontal Ataxia in Childhood
}

\section{E. Erasmus ${ }^{1}$ \\ T. Beems ${ }^{2}$ \\ J. J. Rotteveel ${ }^{1}$}

\section{Abstract}

Frontal ataxia may be the result of a unilateral frontal lesion. In this report three cases are presented with ataxia due to right frontal lesions. One case concerns a boy presenting with an unsteady gait and titubation of the trunk, mimicking developmental disequilibrium and with complex partial seizures. It proved to be caused by a small right-sided cavernoma in the middle frontal gyrus. After surgical intervention the symptoms and the seizures disappeared. Two subsequent cases concern teenage patients presenting with headache after an ENT infection and on physical examination mild dysmetric function of the upper limbs and slight disequilibrium, due to right-sided frontal lobe abscesses. After neurosurgical and antibiotic therapy the symptoms were relieved. The frontal origin of ataxia should be considered in children presenting with a "cerebellar syndrome". Frontal gait disorders consist of a clinical pattern of different gait disorders. The syndrome has been mentioned in the literature under different names. Our patients show signs compatible with the term frontal disequilibrium, a clinical pattern of frontal gait disorder. This assumes walking problems characterized by loss of control of motor planning, leading to imbalance. Remarkably, frontal ataxia may mimic developmental delay as demonstrated in the first case and may be the leading mild symptom in extensive frontal lobe damage as demonstrated by the two other cases. We suppose that frontal ataxia is the result of a disturbance in the cerebellar-frontal circuitries and an impairment of executive and planning functions of the basal ganglia-frontal lobe circuitry.

\section{Key words}

Childhood $\cdot$ frontal lobe $\cdot$ ataxia

\section{Introduction}

Gait ataxia or frontal lobe ataxia is a well known clinical entity in neurological practice. The stance in gait ataxia is wide based, the trunk upright, gait is unsteady, and falls are common. There are no sensory or other cerebellar symptoms. Often medial frontal lobe dysfunction, especially medial bifrontal damage, is responsible. Occasionally a unilateral frontal pathology may cause gait disturbances in childhood.

In this report this is illustrated by the description of a 2-year-old boy presenting with unsteady gait and titubation of the trunk and head due to a small unilateral frontal cavernoma and the history of two teenage patients with a mild ataxic dysfunction caused by a unilateral frontal lobe abscess.

\section{Case Reports}

\section{Patient 1}

This two-year-old boy started walking independently at 18 months. Birth and gestation were unremarkable. He was the firstborn. The parents were non-consanguineous. His mother noticed from the beginning an unsteadiness of gait and a titubation of the head without a tendency of improvement. He often fell down. His speech development was mildly delayed. Otherwise the medical history was unrevealing. On paediatric physical examination no abnormalities were noted. On neurological examination an ataxic gait disturbance and brief periodic staring spells with eyelid contraction without any oculomotor deficits were noticed. Deep tendon reflexes were normal. Laboratory investi-

Affiliation

${ }^{1}$ Department of Paediatric Neurology, University Medical Centre St. Radboud, Nijmegen, The Netherlands

2 Department of Neurosurgery, University Medical Centre St. Radboud, Nijmegen, The Netherlands

Correspondence

C. E. Erasmus MD · Department of Child Neurology · University Medical Centre St. Radboud · PO Box 9101 6500 HB Nijmegen · The Netherlands·E-mail: c.erasmus@cukz.umcn.nl

Received: May 2, 2004 · Accepted after Revision: September 12, 2004 

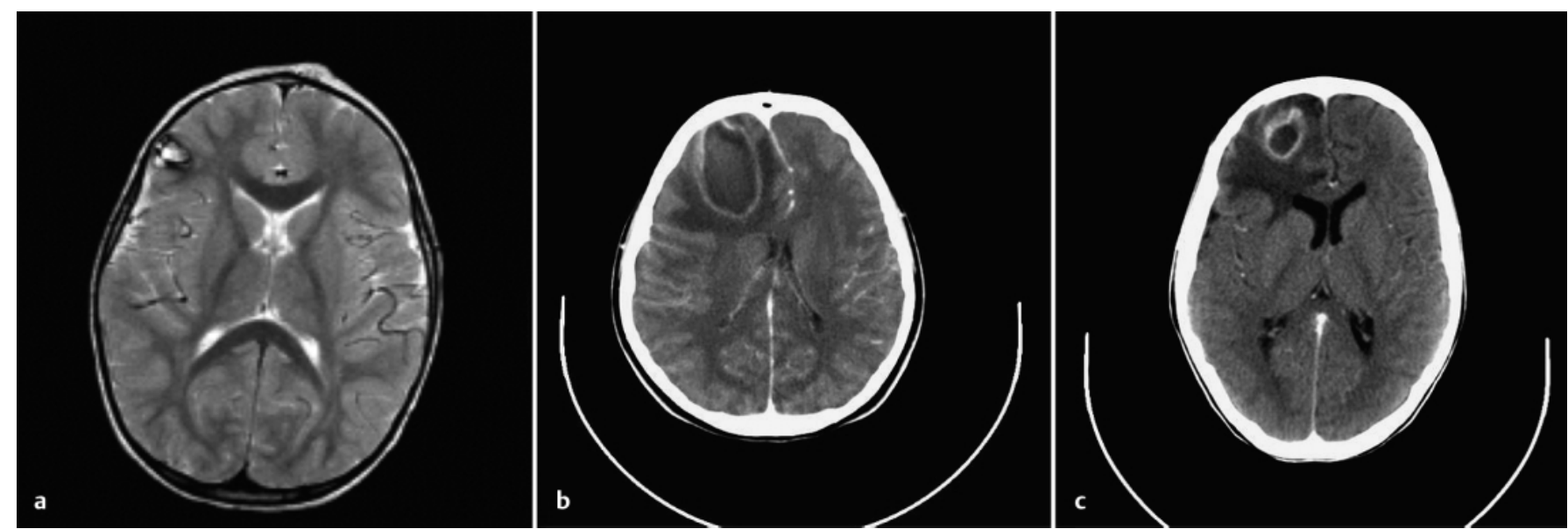

Fig. 1 a to c a Cerebral MRI, $T_{2}$-weighted image of Patient 1. Before craniotomy. A small lesion compatible with a cavernoma is seen in the right middle frontal gyrus. $\mathbf{b}$ Preoperative cerebral CT of Patient 2 after contrast administration showing an contrast-enhancing lesion in

gations revealed no abnormalities on the blood and CSF examinations. Metabolic screening was normal. A cerebral MRI examination revealed a small cavernoma in the medial frontal gyrus on the right side (Fig. 1a). An EEG showed right-sided frontal hypofunctional activity with frequent prefrontal low-voltage spikes.

The patient underwent a minimally invasive, navigated frontal craniotomy with extirpation of the right frontal cavernoma. There were no complications. Immediately afterwards he was able to walk independently without falling. The seizures disappeared.

\section{Patient 2}

This 13-year-old girl started complaining of headaches six weeks before admission. An ENT surgeon diagnosed a nose septum abscess requiring drainage and a 2-week-treatment with antibiotics. Two days before admission the headaches worsened. She had always been a healthy girl. There was no history of trauma, drug abuse or seizures. The family history was unremarkable. She attended high school. She used an analgesic (paracetamol) at that time. Medical and physical examinations were normal. She was not febrile. On neurological examination she showed no signs of raised intracranial pressure. No abnormalities except for slight disequilibrium and a discrete dysmetric function of the left upper limb with mild titubation of the trunk were seen. A cerebral CT with contrast enhancement revealed a right-sided $3 \times 5 \mathrm{~cm}$ large abscess in the upper frontal gyrus, surrounded by edema (Fig. $1 \mathbf{b}$ ). There was a shift of the midline. There were no signs of sinusitis. Blood examination showed no signs of an active infection. The neurosurgeon removed $25 \mathrm{~mL}$ of pus with burr-hole drainage. Streptococcus milleri was cultured. Appropriate high doses of antibiotics were administered intravenously for 6 weeks. The dysmetric dysfunction disappeared soon after surgery. The balance normalized.

\section{Patient 3}

This healthy, 10-year-old boy experienced a subacute throbbing headache accompanied by a brief period of difficulty with speaking and an unsteady gait. One month prior to admission he expe- the right upper frontal gyrus, very suspect for an intracerebral abscess. c Preoperative cerebral CT of Patient 3 showing an intracerebral abscess in the right upper frontal gyrus.

rienced an inflammation of the middle ear. He remained tired, had periodic headaches and episodes of nausea following the ear inflammation. He frequently required analgesics (paracetamol). On admission he was ill, but had no fever. No signs of shock were present. On neurological examination he was restless and impaired in verbal communication. No cranial nerves, motor or sensory dysfunctions were noticed. A slight disequilibrium existed and reaching reactions showed mild dysmetric features. Stretch reflexes were normal. However, at the left side he showed a Babinski reflex. He refused to walk.

The cerebral CT after contrast administration showed a large, right-sided frontal abscess surrounded by edema and accompanied with midline shift (Fig.1 c). The inflammatory parameters in blood were normal. Fifty $\mathrm{mL}$ of pus were drained by burr-hole drainage and Streptococcus pneumoniae was cultured. Postoperatively he went on a 6-week-regime of high dose intravenous antibiotic treatment. Recovery was uncomplicated and the symptoms of disequilibrium and dysmetria disappeared over several days.

\section{Discussion}

Cerebellar and sensory ataxia are well delineated clinical phenomena. Gait ataxia or frontal lobe ataxia is less well known. The neuronal circuitry for motor planning and balance regulation contains the projections between the frontal premotor regions and the pontocerebellar areas and between the basal ganglia and supplementary motor area (SMA) $[4,5,10]$.

Not only primary frontal damage, but also a lesion in the corticocerebellar pathways, corticocortical fibers and basal ganglia/ frontal lobe circuitry may impair motor activation.

The precise localization within the frontal lobe of the dysfunctional generator resulting in the described symptoms is unknown [2]. Bilateral lesions in the medial part of the frontal lobes are most often reported to be responsible for gait ataxia, in 
particular, substantial lesions in the SMA [2,3]. On the other hand, Sunohara et al described an adult patient with the phenomenon of gait ataxia with only unilateral vascular damage in the inferior and middle frontal gyri [9]. Bennett et al described three pediatric patients presenting with episodic or progressive gait ataxia due to "silent" irritative activity. The EEG showed slow spike and wave discharges in the bifronto-temporal regions in the presence of normal occipital activity. The ataxia disappeared after administration of antiepileptic drugs [1].

Takakusaki et al described a two-year-old boy with a unilateral demyelinating lesion in the white matter adjacent to the right frontal horn resulting in subacute episodes of ataxic gait [11]. Sibon et al reported in a review that in adults focal lesions in the basal ganglia and frontal subcortical white matter can produce a frontal gait disorder mimicking parkinsonism $[7,8]$.

Gait ataxia has been described in association with different etiological entities, especially with structural lesions such as cerebral tumors (gliomas), abscesses, traumas and strokes [2]. The syndrome of gait ataxia has been named differently by different authors [2]. Our first patient presented with a developmental gait disorder and titubation with abrupt falls. Patient 3 had obvious trunk imbalance, moving from one side to the other, on the other hand patient 2 had no walking problems, but a minimal dysmetric function of the left arm and mild titubation of the trunk. All three patients had a unilateral frontal lesion and showed clinical signs compatible with the term frontal disequilibrium. There is impairment in equilibrium and postural reflexes and the presence of mild signs of upper limb ataxia. Frontal gait disorders consist of a clinical pattern of various gait disorders. Frontal disequilibrium is one clinical pattern. Others mimic parkinsonism, a particular form of gait disorder is the syndrome of gait ignition failure (previous called "lower half parkinsonism", Marche à petits pas) [6-8].

The frontal abscesses caused visible extensive frontal lobe damage and frontal ataxia seems likely. A unilateral, small frontal lesion in a young child can cause a frontal lobe dysfunction, especially when the lesion gives rise to epileptic activity.

Focal cortical lesions can cause irritative dysfunction which may spread to the contralateral frontal region and can therefore cause frontal disequilibrium and bilateral ataxic signs, especially in childhood. The substantial frontal dysfunction can be demonstrated by EEG registrations.

In conclusion, frontal disequilibrium has its own specificity. In a toddler it may mimic a developmental motor disorder. It should be included in the differential diagnosis of ataxia in childhood. In the assessment of ataxia frontal lobe lesions must be excluded.

\section{References}

${ }^{1}$ Bennett HS, Selman JE, Rapin I, Rose A. Nonconvulsive epileptiform activity appearing as ataxia. Am J Dis Child 1982; 136: 30-32

2 Della Sala S, Francescani A, Spinnler H. Gait apraxia after bilateral supplementary motor area lesion. J Neurol Neurosurg Psychiatry 2002; 72: 77-85

${ }^{3}$ Gentilucci M, Bertolani L, Benuzzi F, Negrotti A, Pavesi G, Gangitano M. Impaired control of action after supplementary motor area lesion: a case study. Neuropsychologia 2000; 38: 1398 - 1404

${ }^{4}$ Herrero MT, Barcia C, Navarro J. Functional anatomy of thalamus and basal ganglia. Child's Nervous System 2002; 18: 386 - 404

${ }^{5}$ Leiner HC, Leiner AL, Dow RS. The human cerebro-cerebellar system: its computing, cognitive, and language skills. Behavioural Brain Research 1991; 44: $113-128$

${ }^{6}$ Nutt JG, Marsden CD, Thompson PD. Human walking and higher-level gait disorders, particularly in the elderly. Neurology 1993; 43: 268 279

${ }^{7}$ Rubio FA. Gait disorders. Neurologist 2002; 8: 254-262

${ }^{8}$ Stolze H, Kuhtz-Buschbeck JP, Drücke H, Jöhnk K, Deuschl G. Comparative analysis of gait disorder of normal pressure hydrocephalus and Parkinson's disease. J Neurol Neurosurg Psychiatry 2001; 70: 289 297

9 Sibon I, Fenelon G, Quinn NP, Tison F. Vascular parkinsonism. J Neurol 2004; $251: 513-524$

${ }^{10}$ Sunohara N, Mukoyama M, Mano Y, Toyoshima E, Satayoshi E. [Frontal pseudoataxia, discussion on its mechanism] (in Japanese). Rinsho Shinkeigaku 1981; 21: 671-681

${ }^{11}$ Takakusaki K, Habaguchi T, Ohtinata-Sugimoto J, Saitoh K, Sakamoto T. Basal ganglia efferents to the brainstem centers controlling postural muscle tone and locomotion: a new concept for understanding motor disorders in basal ganglia dysfunction. Neuroscience 2003; 119: 293 308

12 Vergani MI, Reimao R, Silva AM, Muskat M, Esposito S, Diament A. Multiple sclerosis with early childhood onset. A case report. Arq Neuropsiquiatri 1988; 46: 195-197 